\title{
Gamificando la Evaluación: Una Alternativa a la Evaluación Tradicional en Educación Primaria
}

\section{Gamifying Evaluation: An Alternative to Traditional Evaluation in Primary Education}

\author{
Sergio Tirado-Olivares *, José Antonio González-Calero, Ramón Cózar-Gutiérrez y Rosa M. Toledano \\ Universidad de Castilla-La Mancha, España
}

\section{DESCRIPTORES:}

Gamificación

Evaluación

Educación primaria

Ciencias naturales

Diario de clase

\begin{abstract}
RESUMEN:
En la actualidad, es cada vez más habitual la incorporación de acciones educativas de carácter lúdico a los procesos de aprendizaje, entre los que destaca especialmente la gamificación. A pesar de ello, con frecuencia en estos proyectos no se presta la suficiente atención a la evaluación del aprendizaje producido durante el propio proceso de enseñanza; recurriéndose normalmente a sistemas de evaluación sumativos, como son, por ejemplo, los tradicionales exámenes, al finalizar el proyecto. En el presente estudio pre-experimental, a partir de una metodología mixta y en el contexto de una unidad didáctica gamificada con 24 alumnos de $6^{\circ}$ curso de Educación Primaria en la asignatura de Ciencias Naturales, se evalúa el potencial de un sistema de evaluación formativo gamificado a través del uso de puntos de experiencia y de la renovación tecnológica del tradicional diario de clase o cuaderno del profesor. Los resultados evidencian que este sistema es efectivo tanto para la evaluación del rendimiento académico, como para la evaluación de aspectos educativos transversales como son el trabajo cooperativo, el interés en clase o la revisión de tareas diarias. Además, los resultados revelan que el alumnado muestra un alto nivel de motivación con la dinámica gamificada implementada durante el estudio.
\end{abstract}

\section{KEYWORDS:}

Gamification

Evaluation

Primary education

Natural science

Class diary

\begin{abstract}
:
Nowadays, the incorporation of educational actions with a playful nature into learning processes is increasingly common; among them, gamification stands out. Nonetheless, these projects often do not pay enough attention to the assessment of the learning produced during the teaching process itself; normally resorting to summative evaluation systems, such as, for example, the traditional exams, at the end of the project. In the present pre-experimental study, based on a mixed methodology and in the context of a gamified didactic unit with 24 pupils in 6th grade of Primary Education in the Natural Science subject, the potential of a gamified formative evaluative system is evaluated. This system is composed of the use of experience points and the traditional class diary, or teacher's notebook, as an evaluation tool technologically renewed. The results show that the system is effective for the evaluation of both academic performance and transversal educational aspects such as the cooperative work, the interest in class or the monitoring of daily students' work. In addition, pupils show a high level of motivation with the gamified dynamics implemented during the study.
\end{abstract}




\section{Revisión de la literatura}

La educación no puede ser ajena a los cambios socioculturales que se producen en la sociedad (Díaz y Troyano, 2013). Según la ley educativa (LOMCE, 2013), la educación debe formar a las personas para ser socialmente competentes y conseguir su inclusión plena en la actual sociedad de la información y la comunicación. Por este motivo, los docentes han adquirido la enorme responsabilidad de adaptar sus metodologías y recursos a estos cambios con el propósito principal de mejorar los procesos de enseñanza-aprendizaje (Ortiz-Colón, et al, 2018). En el ámbito concreto de las ciencias y la tecnología, el alumnado muestra un desinterés generalizado por su estudio (Pečiuliauskiene y Belakoz, 2019). Sus contenidos no les resultan motivantes y, con frecuencia, consideran que las asignaturas de este ámbito son difíciles de comprender (Lyons y Quinn, 2010), traduciéndose en resultados académicos cada vez más negativos como se aprecia en los informes PISA (INEE, 2019). Además, esta situación alarmante se agrava conforme el alumnado transcurre por el sistema educativo (Vázquez y Manassero, 2008). De acuerdo con Toma et al. (2019), este desinterés hacia las ciencias se inicia a partir de cuarto curso de Educación Primaria, afectando al logro académico en esta área, tal y como puede observarse en estudios internacionales como TIMSS-Trends in International Mathematics and Science Study (Martin et al., 2012).

Por ello, se necesita un cambio metodológico drástico en la didáctica de las ciencias que propicie una mejora actitudinal (Solbes, 2011). En este paradigma, diferentes proyectos de carácter lúdico son puestos en práctica bajo el término de gamificación. Estos han obtenido resultados positivos en áreas del conocimiento científico tanto a nivel de rendimiento académico, como a nivel motivacional (Hursen y Bas, 2019; Jagušt et al., 2018). Sin embargo, es habitual que, una vez finalizado el proyecto, el logro académico se continúe evaluando a través de un examen final confeccionado para tal fin. No obstante, la gamificación posibilita nuevos planteamientos evaluativos que deben ser examinados.

\subsection{Gamificación: origen, características y beneficios educativos}

El concepto de gamificación tiene su origen en el ámbito empresarial. Tal y como Feijóo y Hernantes (2017) afirman, su nacimiento surge de la necesidad de "enganchar" a compradores y afianzar lazos hacia los productos de una empresa. Aunque fue en 2002 cuando Nick Pelling acuñó el término gamificación (Marczewski, 2013), no es hasta los últimos años cuando se ha extendido su uso (Bai et al., 2020). De acuerdo con Alsawaier (2018), la gamificación está relacionada con atraer a las personas, resolver problemas y mejorar la motivación por medio de adoptar mecánicas y dinámicas del juego. En esta línea, Kapp (2012) definió la gamificación como la integración de características, mecánicas y la estética de los juegos a contextos no lúdicos con el propósito principal de mejorar la motivación o implicación de los participantes.

Así pues, en las situaciones de enseñanza escolares rápidamente se observó la posibilidad de introducir la gamificación, en cierta medida auspiciado por el papel del juego en el desarrollo cognitivo. Ya Piaget (1952) hablaba de esta importancia del juego en el desarrollo cognitivo del niño. Esto, unido a que las nuevas tecnologías han permitido nuevas posibilidades pedagógicas (Hamari, et al., 2014), fomenta la necesidad de conocer qué ventajas y antecedentes existen en el campo concreto de la educación. Dicho esto, no debe confundirse el concepto de gamificación con el uso del juego puntualmente para impulsar el aprendizaje (Nolan y McBride, 2014), lo que se enmarcaría en la conocida metodología Game Based Learning. De acuerdo con Nistor e Iacob (2018), mientras la gamificación presenta características asociadas a agregar elementos inspirados en el juego a la unidad didáctica, aplicando mecánicas del juego a un entorno no lúdico propiamente, Game Based Learning está relacionado con el uso de juegos para alcanzar resultados de aprendizaje. Por tanto, siguiendo la línea de estos autores, la gamificación del aula no puede lograrse utilizando únicamente juegos comerciales o simuladores virtuales y, por tanto, la evaluación del proceso de aprendizaje tampoco.

Autores como Kapp (2012) resaltan que, entre las ventajas fundamentales, al ser la integración de elementos, mecánica y dinámicas del juego la base, la motivación del alumnado mejora. Partiendo de la premisa de este autor, la monotonía en el aula queda superada por medio de la realización de retos, puntos de experiencia y avatares que impulsan al alumnado a querer participar. Asimismo, la importancia recae en la búsqueda de un objetivo concreto, que simula una situación real y que permite, a través de cómo el alumno afronta los retos, analizar cómo se está produciendo su aprendizaje 
(Zichermann y Cunningham, 2011). Además, este contexto educativo de carácter lúdico es compatible con implementar en el aula metodologías basadas en la cooperación y la resolución de problemas (Díaz y Troyano, 2013; Jagušt, et al., 2018).

Tal ha sido su interés e inclusión en el ámbito educativo que diferentes escuelas ya han implementado proyectos educativos en sus centros basados en gamificación. Proyectos como "Pequeños detectives al rescate" en el CEIP Reino de Murcia (2018), "El compromiso de la galaxia Deitania" en el centro Deitania Comarcal (2018) y la iniciativa en Madrid de "Operación Beijerinck" (2018) son ejemplos claros. Entre estos proyectos se aprecian similitudes básicas como la utilización de avatares, la realización de retos, la plasmación de un objetivo final o el trabajo cooperativo. Estos, pese a mostrar grandes beneficios respecto a cómo se produce el desarrollo de las sesiones, evidencian una carencia común concreta: un proceso o sistema de evaluación viable y eficaz dentro del proyecto de gamificación que sustituya a la tradicional evaluación sumativa a través de examen (Zepeda-Hernández et al., 2016).

\subsection{Modelos tradicionales de evaluación}

De acuerdo con Ascendio Peralta (2016) las metodologías centradas en el profesor como diseminador de contenidos teóricos son, aun actualmente, las más habituales. En este contexto, el alumnado tiene un rol pasivo al ser mero receptor de estos contenidos. Contenidos que, posteriormente, deben plasmarse en un examen. Autores como De Ory y Ruiz (2011) indican que este examen es el instrumento más frecuentemente utilizado por el profesorado para medir el aprendizaje conseguido pero que, pese a su utilidad, no permite el análisis del proceso de adquisición de estos conocimientos por parte del alumnado.

Álvarez Menéndez (2009) indica la necesidad de un cambio de perspectiva en los procesos de evaluación hacia una evaluación formativa: en la que no solo se analice el producto final del aprendizaje, sino también el proceso, posibilitándose así simultáneamente el análisis de las competencias necesarias para aprender. Según el autor, el aprendizaje por competencias se está volviendo cada vez más popular; sin embargo, se hacen necesarios nuevos sistemas evaluativos continuos que posibiliten su análisis de forma eficaz conjuntamente con la adquisición de los conocimientos teóricos esenciales que los alumnos deben aprender. Esta premisa se justifica también por la preocupación mostrada por docentes en formación por tener otros sistemas o instrumentos de evaluación además del examen con el fin de realizar una evaluación lo más completa posible al poder también evaluar lo acontecido diariamente en el aula. Entre estos instrumentos, valoran positivamente el uso del diario de clase o cuaderno del profesorado (López-Lozano y Solís, 2015).

\subsection{Sistemas por puntos y diario de clase en procesos de gamificación}

Una unidad de trabajo gamificada puede incorporar herramientas para fomentar el aprendizaje interactivo de los estudiantes, como Kahoot o Quizizz (Navarro, 2017), pero se necesitan promover sistemas para el seguimiento continuo del aprendizaje logrado y que permitan, a su vez, el análisis de la reflexión crítica y comunicación intragrupo para la resolución de los retos (Labrador y Villegas, 2016). La posibilidad de enriquecer propuestas gamificadas con una evaluación de tipo formativo en proyectos de gamificación puede constituir un salto cualitativo fundamental en la aplicación de estas metodologías, pues tal y como Nicol y Milligan (2006) apuntan, el fomento de la autorregulación por medio de aportar información constantemente al aprendiz sobre su progreso propicia el aprendizaje significativo.

Si se realiza una revisión de gran parte de los juegos actuales, con mucha frecuencia encontramos entre sus dinámicas la utilización de puntos para medir el logro alcanzado y desbloquear mejoras o subidas de nivel (Fiellin et al., 2014), reforzando el interés del usuario por continuar jugando (Prestopnik y Tang, 2015). Así, se evidencia sus ventajas extrapoladas al ámbito educativo (Sedeño, 2010). El uso de estos puntos, insignias y tablas de puntuaciones (PBL, de sus siglas en inglés: Points, Budgets y Leaderboards) se correspondería con la denominada gamificación superficial (Marczewski, 2013). Esta es entendida como el uso de ciertos elementos gamificados, pero sin llegar a utilizar las tres estructuras claves de la gamificación (componentes, mecánicas y dinámicas, propias de una gamificación completa o gamificación profunda (Werbach y Hunter, 2015). Metaanálisis actuales 
sugieren que el tamaño de efecto del uso de PBL se acrecienta cuando se incorporan elementos de la gamificación profunda como pueden ser el trabajo colaborativo o la realización de misiones (Huang et al., 2020).

La supervisión del proceso de aprendizaje durante una metodología gamificada puede lograrse gracias a puntos asociados a cada reto (Chou, 2019). Los puntos permiten la regulación del proceso en diferentes niveles capaces de ser alcanzados por el alumnado gracias al correcto desempeño y, además, fomentan el espíritu participativo y motivación por querer superarse (Kapp, 2012). Se ha demostrado que el uso de puntos de experiencia mejora en el alumnado la concentración, comportamiento y disfrute durante la tarea (Wang y Lieberoth, 2016).

Sin embargo, la investigación educativa ha prestado escasa atención a cómo articular en el aula esta monitorización del aprendizaje con fines evaluativos. Continuamente se habla de la necesidad de formar al docente en nuevos recursos tecnológicos (Wu et al., 2019), pero existen pocos estudios sobre la utilización renovada de recursos tradicionales como puede ser el diario, o cuaderno del profesor, pese a obtener una gran acogida por el profesorado, pues su manejo les resulta fácil y cómodo mediante sus ordenadores o tablets (Jiménez-Gómez, 2019). Igualmente, su implementación potencia la retroalimentación efectiva de lo que está aconteciendo en el aula (Constantine y Jung, 2019). Estos diarios de clase permiten la recopilación continua de la observación directa llevada en clase, consiguiéndose el objetivo buscado de la evaluación formativa (Porlán, 2008; Sá, 2002). Estos beneficios, tras adaptar esta herramienta pedagógica al formato digital, se han apreciado también dentro del campo concreto de la didáctica de las ciencias (Paek y Fulton, 2017). El uso de cuadernos digitales del profesor ha dado lugar a la creación de aplicaciones específicas que sustituyan al diario de clase convencional como Additio o IDoceo (Jiménez-Gómez, 2019), pero no existe literatura científica que demuestre la eficacia de su uso dentro de proyectos gamificados.

Por consiguiente, puesto que para alcanzar una experiencia educativa de éxito se necesita una base científica que la sustente (Flecha y Molina, 2015), se requieren más estudios que analicen la interconexión entre metodologías gamificadas y sistemas de evaluación formativos. Así, el presente estudio persigue estudiar el potencial de un sistema de evaluación formativo gamificado (SEFG) para sustituir instrumentos de evaluación tradicionales. En concreto, se plantean los siguientes objetivos:

- Evaluar el potencial de la evaluación del aprendizaje ofrecida por el SEFG en comparación con la evaluación sumativa con estudiantes de $6^{\circ}$ curso en la materia de Ciencias Naturales.

- Analizar la eficacia del SEFG en comparación con la evaluación sumativa para evaluar aspectos transversales de la dinámica de clase tales como el trabajo cooperativo, comportamiento e interés y el seguimiento del trabajo realizado por el alumnado.

- Conocer la percepción del alumnado sobre el uso de la gamificación como metodología de enseñanza.

\section{Método}

\section{Diseño de investigación}

Para analizar el SEFG se desarrolló una investigación mixta pre-experimental. Los 24 alumnos (14 chicas y 10 chicos) de una clase de $6^{\circ}$ curso de Primaria de un colegio urbano de la región de Castilla-La Mancha participaron en el proyecto. El tipo de muestreo utilizado es no probabilístico y de conveniencia debido a las facilidades encontradas para llevar a cabo la intervención tanto en el centro educativo como en el grupo participante. Este proyecto se extendió a lo largo de 14 sesiones con una hora de duración cada sesión. El número de sesiones se correspondió con la planificación prevista para una unidad didáctica sobre nutrición del área de Ciencias Naturales.

Esta unidad se desarrolló mediante una metodología gamificada, a pesar de lo cual la profesora responsable del grupo mantuvo los instrumentos de evaluación sumativos establecidos, que comprendían, por ejemplo, la realización de un examen al finalizar la unidad. Sin embargo, en esta 
ocasión además el investigador a cargo del proyecto llevaba una evaluación paralela recogiendo los datos del proceso de aprendizaje acontecidos diariamente. Estos datos, o puntos de experiencia gamificados, eran anotados en un diario de clase. De este modo, las variables que conforman el presente estudio son, por un lado, la efectividad del SEFG para medir el proceso de aprendizaje en relación con los instrumentos que son implementados habitualmente y, por otro lado, cómo la inclusión de esta dinámica gamificada influye en el interés del alumnado.

\section{Instrumentos de recogida de datos}

En esta investigación, el instrumento de recogida principal de datos es, simultáneamente, elemento principal de análisis; pues se quiere conocer la eficacia del diario de clase digital como herramienta evaluativa. Junto a él, el examen de la unidad y un test mixto motivacional para conocer la percepción del alumnado sobre el proyecto, forman los tres instrumentos que habilitan el análisis de los objetivos de investigación previamente descritos.

El diario de clase fue confeccionado a través de diferentes páginas en formato digital mediante la aplicación de Microsoft Excel. Está diseñado para que los docentes puedan recopilar la información necesaria sobre lo que sucede continuamente en el aula durante el transcurso de la clase. Las hojas para cada sesión están compuestas de tres secciones:

Sección 1. Elementos curriculares. Aunque se trate de un proyecto gamificado, el principal objetivo pedagógico debe mantenerse intacto: fomentar el aprendizaje del alumnado cumpliendo los elementos curriculares que regía la ley de educación (LOMCE, 2013). Por ello, durante el proyecto, cuatro elementos debían ser cumplimentados en esta sección: objetivos didácticos de la sesión, competencias trabajadas, criterios de evaluación y estándares de aprendizaje evaluados. Estos elementos debían cumplirse de acuerdo con la programación didáctica de la asignatura.

Sección 2. Puntuaciones obtenidas. A través de una tabla automatizada, se habilitaba un espacio para recopilar las puntuaciones obtenidas de cada alumno de forma individualizada y por destrezas. Cada alumno obtenía puntuaciones en cinco apartados diferentes: plasmación de conocimientos adquiridos, intervenciones fundadas realizadas en clase, habilidades para la resolución de los retos, trabajo cooperativo y comportamiento. El máximo de puntos alcanzables por el alumnado era 600,200 de los cuales únicamente se podían conseguir gracias a la plasmación de conocimientos aprendidos, facilitándose así la comparativa con los resultados obtenidos en el examen. El 50\% de puntos restantes se conseguían a partir de un correcto trabajo cooperativo durante la resolución de las actividades. Su alto porcentaje se debe a que, según la planificación docente, existía un estándar referido a este aspecto que debía evaluarse extensamente durante la unidad. Los puntos restantes permiten el análisis de aspectos transversales como son el interés en clase o la participación.

Sección 3. Anotaciones. Dado que durante el aprendizaje se producen elementos reseñables a tener en cuenta, la propia herramienta habilitaba un espacio para que el docente pudiera realizar todas aquellas anotaciones, individuales y/o colectivas, que considerara oportunas.

Además, dado que el objetivo principal es poder analizar el aprendizaje logrado por los alumnos en relación con los contenidos curriculares establecidos, automáticamente se iba cumplimentando una hoja anexa al final del cuaderno con las puntuaciones totales que el alumno iba consiguiendo en cada apartado, y de forma total, a través del sumatorio de puntos de cada sesión. De este modo, al finalizar el proyecto, el docente, sin un requerimiento de trabajo añadido, adquiría las puntuaciones globales alcanzadas durante la unidad didáctica de cada alumno.

Con respecto al examen, este fue confeccionado ad hoc para evaluar los contenidos curriculares de acuerdo con el actual currículum de Educación Primaria de Castilla-La Mancha (Decreto 54/2014). El examen había sido diseñado previamente al proyecto al igual que el resto de los exámenes que el alumnado debía realizar a lo largo del curso académico. Este estaba compuesto por diez preguntas que evaluaban los estándares de aprendizaje de la unidad relacionados con la adquisición de conocimientos (Cuadro 1). Este test, corregido por el propio profesorado a cargo de la asignatura, sirvió para realizar la comparativa con el SEFG en relación al logro académico alcanzado por el alumnado. 
Cuadro 1

Examen de la unidad y estándares de evaluación asociados

La función de nutrición, aparatos asociados y sus órganos

Identifica y localiza los principales órganos implicados en la realización de las funciones vitales del cuerpo humano: nutrición (aparatos respiratorio, digestivo, circulatorio y excretor)

Completar una tabla a través de identificar el órgano correspondiente a partir de su función y viceversa: explicar la función de un órgano referenciado en la tabla.

Identifica y describe las principales características de las funciones vitales del ser humano.

Explicar qué es y qué función tiene el aparato digestivo a partir de las siguientes palabras: alimentos, tubo digestivo, digestión, nutrientes, glándulas digestivas

Identifica las principales características de los aparatos respiratorio, digestivo, circulatorio y excretor y explica sus principales funciones.

Identificar los órganos principales del aparato respiratorio en un dibujo

Responder a la siguiente pregunta: ¿qué aparato está representado en la pregunta anterior y cuál es su función? ¿Cuál es el músculo esencial para los movimientos respiratorios? Explica su función.

Responder a la siguiente pregunta: ¿Cuál es la función del aparato circulatorio? Y explicar brevemente su funcionamiento

Contestar a las siguientes preguntas sobre el aparato excretor. ¿Qué es la excreción? ¿Qué elementos forman el aparato excretor?

\section{Hábitos de vida saludables}

Reconoce estilos de vida saludables y sus efectos sobre el cuidado y mantenimiento de los diferentes órganos y aparatos.

Identifica y valora hábitos saludables para prevenir enfermedades y mantiene una conducta responsable

Unir con flechas diferentes hábitos de salud con su posible consecuencia y con el aparato relacionado.

Conoce y explica los principios de las dietas equilibradas, identificando las prácticas saludables para prevenir y detectar los riesgos para la salud

Observa, identifica y describe algunos avances de la ciencia que mejoran la salud (medicina, producción y conservación de alimentos, potabilización del agua, etc.).

Responder a las siguientes preguntas justificando su respuesta. ¿Se pueden formar nuevos tejidos o reparar tejidos dañados? ¿Qué avances existen para que todo ser humano disponga de agua potable? ¿Qué avance ha ayudado a prevenir el consumo de alimentos en malas condiciones?

Reconoce los efectos nocivos del consumo de alcohol y drogas

Identificar afirmaciones que sean falsas relacionadas con el consumo de alcoholy drogas razonándose la respuesta.

Identifica y adopta hábitos de higiene, cuidado y descanso.

Identificar hábitos saludables: ¿Qué hábitos saludables tienes? Escribe tres y explica por qué son importante

Por último, tras el proyecto se requirió al alumnado que cumplimentara el test motivacional para analizar su percepción de la dinámica gamificada implementada. Este cuestionario, confeccionado ad hoc, analizaba aspectos concretos de la gamificación utilizados durante el proyecto y permitía, a través de diferentes ítems, la comparativa de esta metodología con la dinámica habitual que el grupo seguía en el aprendizaje de Ciencias Naturales (Figura 1). El diseño del instrumento permitía valorar la preferencia del alumnado por una u otra metodología o la indiferencia ante ambas opciones. Además, el cuestionario habilitaba un espacio para que el alumnado expusiera su visión personal sobre qué les había parecido el proyecto, así como cuáles eran, en su opinión, los elementos gamificados más interesantes o qué actividades han sido de mayor agrado personal. Las indicaciones sobre cómo cumplimentar el test fueron realizadas en los minutos previos a su administración a los participantes. 
Figura 1

Test motivacional cumplimentado por el alumnado

iHola chicos! ¿Qué pensáis de este tipo de clases?

\begin{tabular}{|l|l|l|l|}
\hline Las clases de este tema me han resultado más interesantes que las de temas anteriores & Si & Igual & No \\
\hline Las actividades hechas en clase y la actitud del profesor me han mottuado a aprender & & \\
\hline Estas clases me han servido para entender mejor los contenidos del tema & & \\
\hline Clases asi me mottuan a aprender sobre este tema y sobre las clenclas en general & & & \\
\hline Utellyar instrumentos cientificos y hacer experimentos me qustan y me ayudan a aprender & & & \\
\hline Me gusta más aprender con actividades colaborativas que siguiendo el libro & & \\
\hline
\end{tabular}

Cuéntame un poco qué te han parecido las clases: qué es lo que más te ha gustado, qué cambiarías...

\section{Condiciones gamificadas del proyecto}

Para el desarrollo del estudio, previamente a la implementación del proyecto se confeccionó una página web para desarrollar la secuencia de enseñanza gamificada. Esta página web servía de hilo conductor del proyecto. En ella se plasmaban todos los retos que el alumnado debía realizar, al igual que la narrativa (storytelling) que dotaba de sentido de cohesión y progresión a todas las sesiones de la unidad: el alumnado había despertado en algún lugar del aparato digestivo y debía conseguir escapar de allí (Figura 2). Esta narrativa fomentaba la participación del alumnado a través de la escucha activa al presentar preguntas o huecos a resolver durante su lectura.

\section{Figura 2}

Captura de la página web creado para el proyecto de la sesión 7
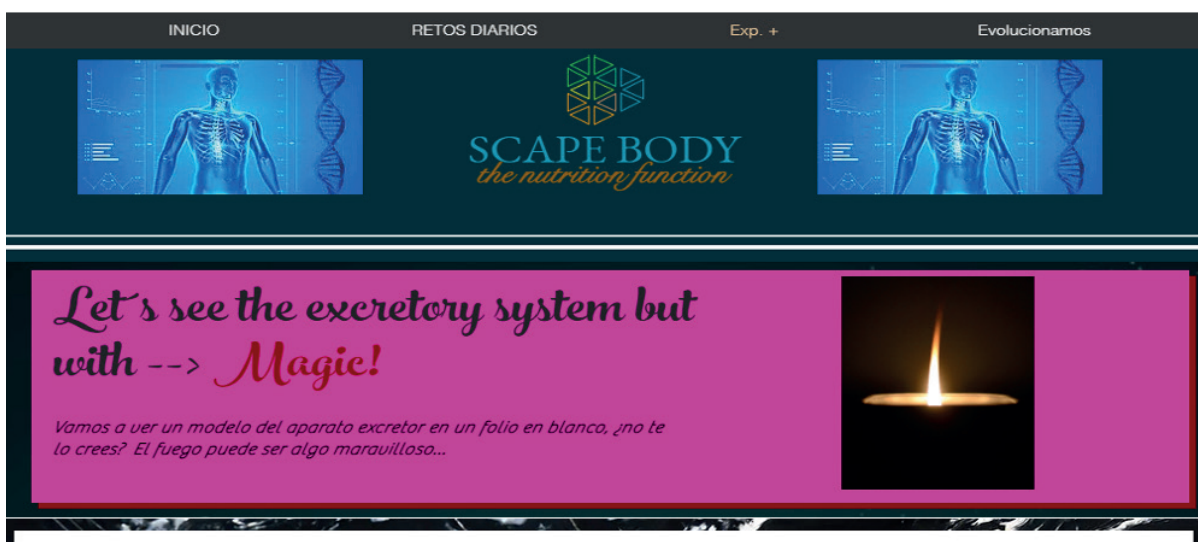

El aparato excretor... una solución casi perfecta

Cuando parecía claro que esta vez sí que sí, que a través de _____ saldriamos al exterior con el resto de desechos celulares, tuvo que aparecer Sergio... el profesor de prácticas: ya se podía haber ido a otro centro.

Se ve que el señor Sergio era un poco escrupuloso: I do not go there! the ureters and kidneys smell very bad (Si, encima es profesor de inglés... vaya suerte hemos tenido. \#lronía

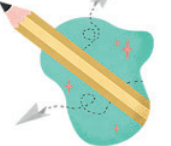


A continuación, en cada sesión se realizaron retos ligados a contenidos de los cinco aparatos que participan en la función de nutrición, pues así sucedía en la historia en la que el alumnado estaba inmerso. Igualmente, a través de esta página web, los alumnos podían ver cada uno de los elementos que integraban las sesiones de aprendizaje, así como los puntos de experiencia que conseguían cada día y el nivel alcanzado debido a estos puntos. Conforme el alumnado progresaba, desbloqueaba un avatar superior que reflejaba un nuevo nivel de conocimiento científico. Con todos estos elementos, se perseguía poder llevar a cabo una gamificación profunda del aula siguiendo las recomendaciones obtenidas del metaanálisis de Huang et al. (2020).

Basándonos en el esquema de Werbach y Hunter (2015), este proyecto englobaba las tres estructuras básicas que debe constituir un proyecto de gamificación profunda: los componentes gracias a los avatares y puntos de experiencia, las mecánicas por medio de los retos, la retroalimentación y cooperación y las dinámicas a través del sentimiento de progresión e historia narrativa subyacente. Así, durante todas las sesiones, el papel tanto del alumnado como del profesor (investigador) fue invariante (Figura 3).

Figura 3

Organigrama de las sesiones y funciones a desempeñar por el alumnado y el profesorado

\section{Dinámica de clase durante las 14 sesiones del proyecto}

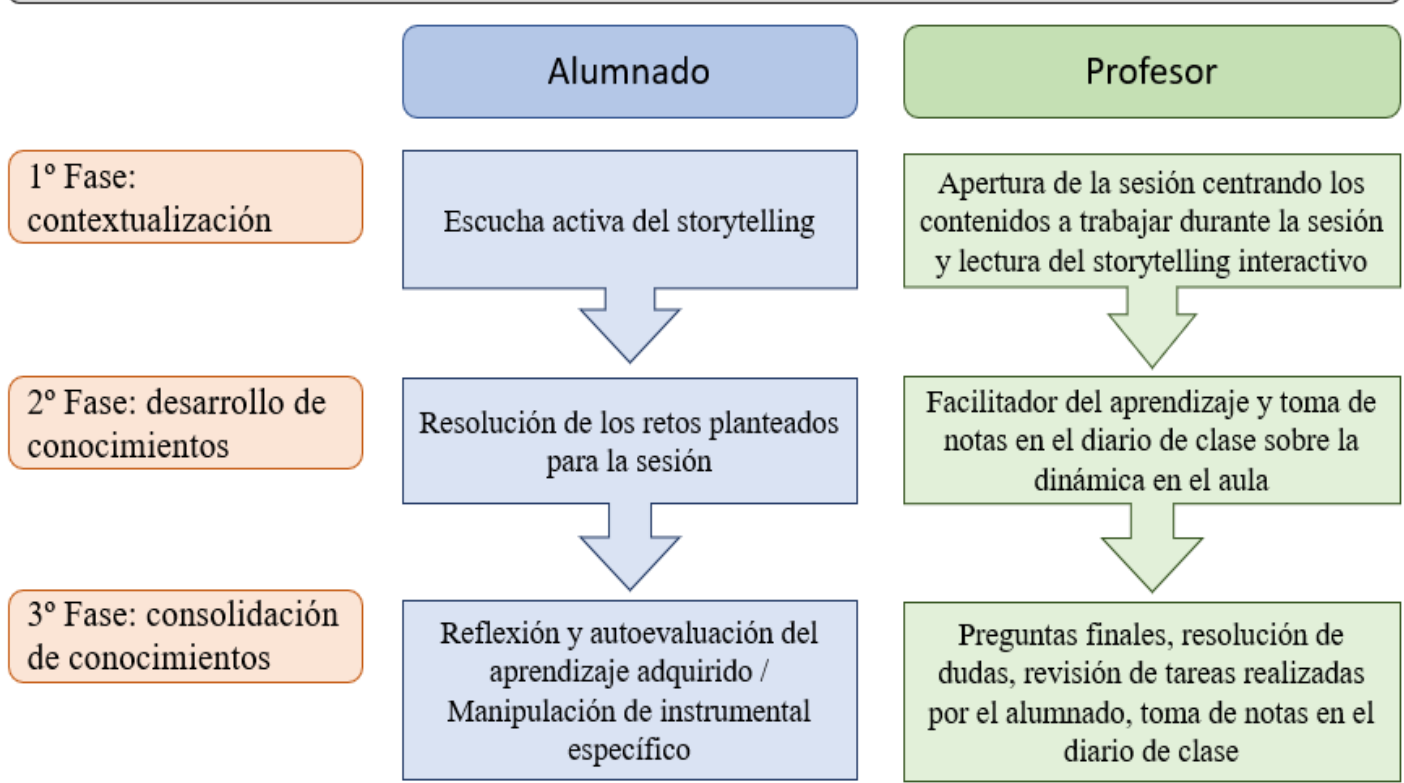

Cada sesión comenzaba con una contextualización a través del storytelling que situaba al alumnado en los contenidos curriculares a trabajar ese día y que además les incentivaba a trabajarlos (Rowcliffe, 2004). Posteriormente, la siguiente fase, de desarrollo de contenidos, era diferente cada día, pues los retos a desarrollar eran variados: búsqueda de pistas, apertura de cajas con candados de combinación alfanuméricos o símbolos, búsqueda de información a través de linternas UV para revelar escritos invisibles, trabajo con instrumental del ámbito científico, etc. Así, se fomentaba el trabajo de contenidos curriculares a través de retos ligados a puntos de experiencia que permitían, a su vez, la recopilación de estos puntos. Con frecuencia, estos retos eran desarrollados de forma cooperativa en grupos de cinco personas; lo que permitía el fomento de competencias claves y estándares de aprendizaje relacionados al respeto como son la capacidad de trabajar con otros y alcanzar soluciones conjuntamente de forma dialogada. Un ejemplo de estos retos es visible en la Figura 4. 


\section{Where are we? --> The road starts}

Hemos aparecido en alguna parte del sistema digestivo, pero no sabemos en cuál exactamente.

Cuando hayáis realizado cada una de las tareas, enseñarsela al profesor para que os dé la puntuación obtenida y os ponga el sticker correspondiente

1. A partir de la plantilla en blanco, hacer un esquema del aparato digestivo con los órganos vistos anteriormente (colocando su nombre en inglés y castellano) e identificar cuáles son partes del tubo digestivo y cuáles glándulas digestivas. $(5 \times$ p.)

2. Coloca estos elementos en el órgano correspondiente, de este modo, el reto de tener un modelo completo habrá sido conseguido y seremos capaces de saber qué función desempeñan cada uno con mayor facilidad. ( 5 xp; 1 por cada uno bien colocado)

- Dientes (caninos, incisivos, premolares y molares)

- Pliegues o vellosidades

- Váluulas: cardias y el píloro

- La lengua y las glándulas salivares

- El ciego, el colon y el recto

3. Coloca la función correspondiente a cada órgano, aquél que quede sin función es donde nos encontramos. (16 xp; 2 por cada función bien explicada)

- Se forma el bolo alimenticio

- En él se produce la absorción intestinal

- Se elabora el jugo pancreático

- Une la faringe con el estómago

- Conduce los alimentos hacia el esófago

- Transforma en heces las sustancias que el cuerpo no aprovecha

- Produce bilis

- Por él se expulsan los residuos al exterior

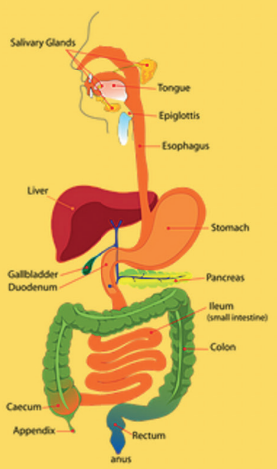

4. ¿Ya sabemos donde estamos? Estamos en:

Explicar qué función desempeña este órgano. ¿Se te ocurre como podemos explicar la digestión de manera práctica? Después haremos un experimento científico para demostrarlo: ; LAS ENZIMAS ACTÚAN! (15xp; por explicar bien y plantear hipótesis correctas y los otros 10 puntos acorde a la buena realización del experimento).

Cuando hayamos resuelto los cuatro retos y sepamos en qué organo nos encontramos, su nombre en inglés nos dará la combinación para abrir el cofre. Cada letra o grupo de letras corresponde a un número diferente, utilizo solo aquellas combinaciones que aparezcan en su nombre:

$$
\begin{array}{rlll}
m a \rightarrow 0 & c r \rightarrow 1 & 0 \rightarrow 4 & f i \rightarrow 7 \\
s t \rightarrow 2 & p r \rightarrow 5 & e \rightarrow 8 \\
t u \rightarrow 3 & c h \rightarrow 6 & c k \rightarrow 9
\end{array}
$$

Finalmente, en cada sesión existía una actividad de cierre o ampliación de contenidos que permitía realizar una recapitulación de lo aprendido. A modo de ejemplo, en estos últimos minutos de clase se desarrollaron actividades de autoevaluación como juegos temporalizados, Kahoot o concursos, y se manipuló instrumental relacionado a los sistemas estudiados como son los espirómetros o fonendoscopios. Además, en gran parte de las sesiones se requería al alumnado la realización de retos en casa para practicar los contenidos vistos en clase. Dentro de estos, existía la posibilidad de hacer además ciertos retos extra de carácter voluntario para la obtención de más puntos de experiencia.

Así, mientras el alumnado resolvía las actividades planificadas para cada sesión, el investigador tenía el tiempo suficiente para recopilar la información sobre cómo se estaba produciendo el aprendizaje anotando los puntos conseguidos en el diario de clase. La consecución de estos puntos se evaluaba de acuerdo con la capacidad del alumnado por alcanzar la resolución de los retos y a la forma de actuación individual y/o colectiva. Además, continuamente se hacían preguntas de control para verificar el grado de compresión del alumnado. Estas preguntas, tanto de procedimiento como de contenido teórico, también tenían su influencia en el número de puntos alcanzado. Al finalizar la unidad, fue el momento de distribuir los otros dos instrumentos que permitían la evaluación de los objetivos de investigación: el examen de la unidad y el test de motivación.

\section{Análisis posterior de los datos obtenidos}

A través del paquete estadístico SPSS vs.24, se procedió a comparar por medio de un análisis inferencial de correlaciones los puntos conseguidos gracias al SEFG con las calificaciones establecidas por la tutora según la 
evaluación tradicional que realizaba habitualmente mediante la observación directa, la revisión de tareas y las calificaciones obtenidas en el examen final. Igualmente, los datos cuantitativos obtenidos por el cuestionario motivacional fueron triangulados con la parte cualitativa de este. Para esta última parte del análisis se empleó el programa ATLAS.ti. Para el análisis de las respuestas, se implementó el método comparativo constante; el cual permite la comparativa sistemática entre códigos y la elaboración de conceptos a partir de la información obtenida (Taylor y Bogdan, 2000).

\section{Resultados}

Para analizar los dos primeros objetivos de investigación planteados se procedió a comparar el SEFG con el sistema de evaluación tradicional previamente explicado. Así, se ha comparado las dos formas de evaluación planteadas durante el proyecto con respecto a aspectos específicos ligados al aprendizaje como son el rendimiento académico, el trabajo cooperativo, la realización de tareas e interés y comportamiento del alumnado.

Posteriormente, para analizar el tercer objetivo de la presente investigación, se ha analizado la visión del propio alumnado de la gamificación como metodología educativa en comparación con la dinámica habitual de clase. Las respuestas dadas en cada uno de los ítems en el cuestionario de motivación han sido trianguladas con la visión personal mostrada en la pregunta abierta del mismo cuestionario. A continuación, se detallan los resultados obtenidos en conexión con cada uno de los objetivos de la investigación.

\subsection{Relación entre calificaciones del SEFG y el sistema tradicional}

El análisis del potencial del SEFG es objetivo primordial de la presente investigación. Por ello, se ha realizado un análisis estadístico inferencial a través de la correlación de Pearson entre la calificación obtenida por el proceso de evaluación tradicional con los puntos de experiencia totales alcanzados por el alumnado y recogidos en el diario de clase. Este test ha sido utilizado tras comprobar los supuestos de normalidad a través del test de Shapiro-Wilk. Esta prueba reflejó que existe una asociación estadísticamente significativa entre ambos conjuntos de datos: $r=0,96 ; p<0,01$.

Así, podemos afirmar que, con un nivel de significancia muy alto, existe relación entre las puntuaciones obtenidas por los alumnos en el SEFG y la nota obtenida por el sistema de evaluación tradicional. Además, la asociación entre ambas medidas es muy elevada (Hattie, 2009). Esto, además, es visible de forma gráfica (Figura 5).

Figura 5

Recta de regresión obtenida entre las puntuaciones obtenidas por el SEFG y la calificación obtenida en la evaluación tradicional

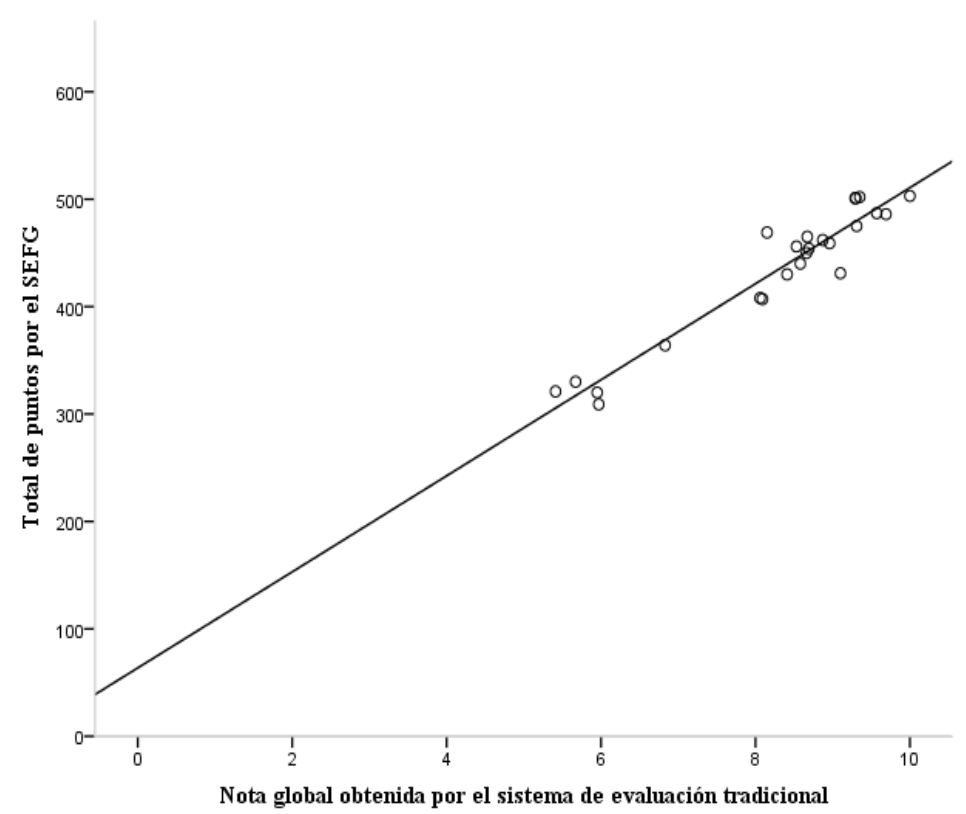


La recta de regresión por mínimos cuadrados evidencia un valor muy cercano a la unidad en su coeficiente de determinación $\left(R^{2}=0,922\right)$. Por tanto, podemos afirmar que existe una evidente asociación entre ambos sistemas de evaluación. El SEFG reporta calificaciones prácticamente idénticas a la obtenidas por el sistema evaluación tradicional constituido por una prueba final y la valoración personal que el docente realiza a través de la observación directa.

\subsection{Análisis de la calificación obtenida en el examen en relación con los puntos obteni- dos referidos a la demostración de conocimientos}

Una vez conocido que existe relación significativa entre ambos sistemas de evaluación de forma global, conocer si existe asociación en el análisis exclusivo del rendimiento académico es el siguiente objetivo que persigue esta investigación. En este caso, dado que durante el proyecto en todas las sesiones se cumplimentaba en el diario de clase un espacio referente a la demostración de conocimientos adquiridos, la comparación entre estas puntuaciones particulares con la calificación obtenida en el examen final podía realizarse de forma directa.

De nuevo, tras comprobar normalidad a través de Shapiro-Wilk, se ha utilizado la correlación de Pearson para comparar ambos conjuntos de datos. En este caso, se ha obtenido el valor de: $r=0,944 ; \mathrm{p}<0,01$. Esta asociación es de nuevo muy alta basándonos en Hattie (2009); algo que vuelve a ser visible de igual modo gracias a la recta de regresión por mínimos cuadrados (Figura 6).

Figura 6

Recta de regresión obtenida entre los puntos referidos a la plasmación de conocimientos teóricos recogidos en el diario de clase y la calificación total obtenida en examen de la unidad

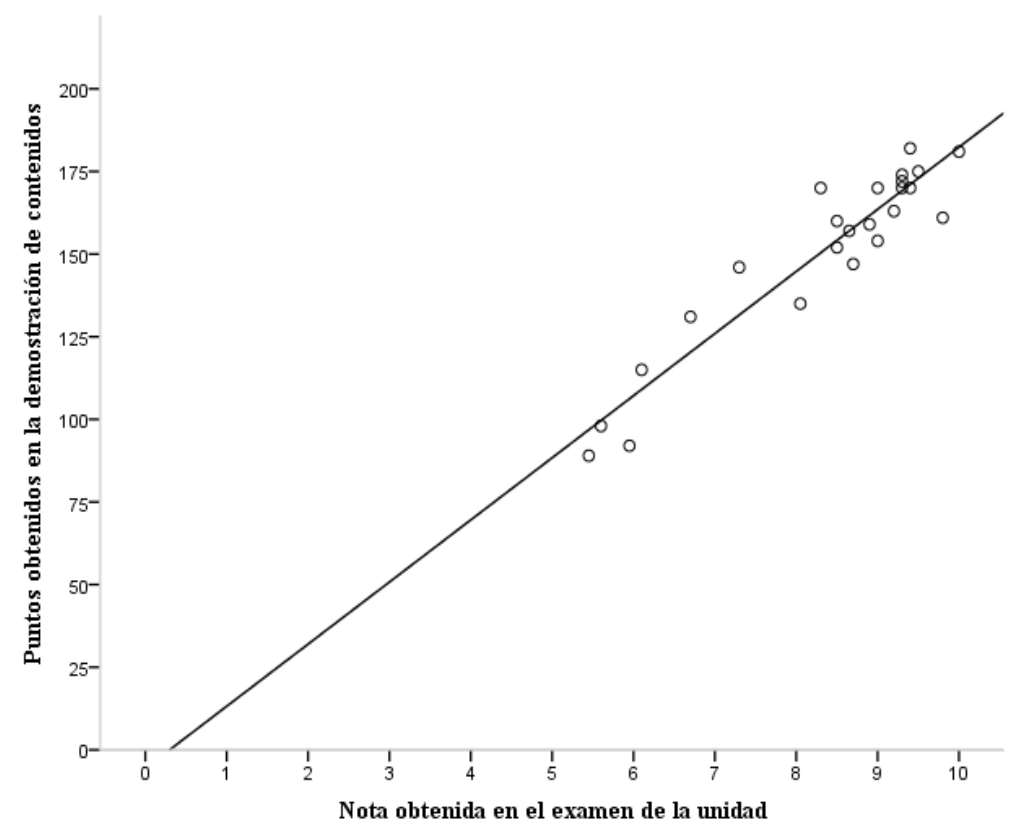

Del mismo modo que sucedía en el caso anterior. El valor obtenido por el coeficiente de determinación se sitúa muy próximo a la unidad $\left(R^{2}=0,891\right)$. Por tanto, centrándonos exclusivamente en el análisis del logro académico, de nuevo ambos sistemas de evaluación reportan niveles de relación significativamente muy altos.

\subsection{Análisis de aspectos acontecidos durante la dinámica de clase}

Siguiendo el esquema de objetivos planteados para esta investigación, el objetivo dos pretende conocer la eficacia del SEFG para la evaluación de aspectos transversales del proceso de aprendizaje como son el trabajo cooperativo, interés y seguimiento de tareas. El análisis de estos aspectos es visible en el Cuadro 2. En este caso, dado que la mayoría de los datos no cumplían con el supuesto de normalidad reportado por la prueba de Saphiro-Wilk, se realizaron correlaciones de Spearman. 
Cuadro 2

Correlaciones de Spearman entre los dos tipos de evaluación sobre aspectos transversales del proceso de aprendizaje

\begin{tabular}{lccc}
\hline & \multicolumn{2}{l}{ Evaluación formativa gamificada } & \\
\cline { 2 - 4 } & Trabajo cooperativo & Comportamiento /interés & Seguimiento de tareas \\
\hline $\begin{array}{l}\text { Evaluación } \\
\text { tradicional }\end{array}$ & $0,755^{* *}$ & $0,750^{* *}$ & $0,761^{* *}$ \\
\hline
\end{tabular}

Nota. ${ }^{* *}$ Las correlaciones son significativas en el nivel $p<0,01$

Con respecto al análisis del trabajo cooperativo, este se incluía dentro de uno de los estándares de aprendizaje a evaluar de acuerdo con la programación. Del mismo modo que ocurría previamente, la correlación de Spearman reporta una elevada asociación entre ambas evaluaciones $(r s=0,755$; $p<0,01)$ siguiendo los valores de referencia aportados por Hattie (2009). Además, se obtuvo en la recta de regresión un valor de $\left(R^{2}=0,685\right)$. Por tanto, podemos afirmar que la evaluación del trabajo cooperativo entre el alumnado puede evaluarse gracias a los puntos de experiencia que cada alumno obtiene en las actividades de trabajo en grupos.

En relación con el comportamiento e interés mostrado por el alumnado, se obtuvieron también correlaciones significativas: $r s=0,750 ; p<0,01$, siendo el tamaño de efecto moderado (Hattie, 2009), de acuerdo al grado de asociación encontrado. En este caso, el coeficiente de determinación obtenido fue de $\left(R^{2}=0,566\right)$. Así pues, se evidencia la relación entre las puntuaciones obtenidas en ambos sistemas.

Finalmente, con respecto al seguimiento de las tareas que el alumnado hace en casa, en el sistema de evaluación tradicional se realizaba únicamente al finalizar la unidad a través de la revisión de las libretas o cuadernos donde los alumnos realizaban sus actividades. Sin embargo, dado que el SEFG estaba diseñado para analizar, además, las intervenciones acertadas realizadas en clase y el interés por realizar de forma eficaz los retos extra, todos estos elementos fueron tomados en consideración. En este caso, la correlación obtenida fue de: $r s=0,761 ; p<0,01$ ), obteniéndose, por tanto, como en los casos anteriores, un tamaño de efecto medio (Hattie, 2009). El valor reportado por el coeficiente de determinación fue de $R^{2}=0,530$.

\subsection{Visión del alumnado sobre el uso de metodologías gamificadas}

Finalmente, conocer la percepción del alumnado permitiría una evaluación del proyecto desde todos los puntos de vista (objetivo 3). Por ello, gracias al cuestionario sobre motivación hacia la gamificación se podría conocer la opinión de los estudiantes partícipes en el proyecto sobre los puntos fuertes que consideran que la gamificación reporta a la dinámica de aula en contraposición a la metodología tradicional habitual de clase que solía implementarse. Estos resultados, son mostrados en el Cuadro 3. 
Cuadro 3

Resultados obtenidos a través del test de motivación

\begin{tabular}{|c|c|c|}
\hline & Sí & Igual / Indiferente \\
\hline $\begin{array}{l}\text { Las clases de este tema me han resultado más interesante que las } \\
\text { clases de temas anteriores. }\end{array}$ & $\begin{array}{l}24 / 24 \\
(100 \%)\end{array}$ & $\begin{array}{l}0 / 24 \\
(0 \%)\end{array}$ \\
\hline $\begin{array}{l}\text { Las actividades realizadas en clase y la actitud del profesor me han } \\
\text { motivado a aprender }\end{array}$ & $\begin{array}{l}22 / 24 \\
(91,67 \%)\end{array}$ & $\begin{array}{l}2 / 24 \\
(8,33 \%)\end{array}$ \\
\hline $\begin{array}{l}\text { Estas clases me han servido para aprender mejor los contenidos de la } \\
\text { unidad }\end{array}$ & $\begin{array}{l}21 / 24 \\
(87,50 \%)\end{array}$ & $\begin{array}{l}3 / 24 \\
(12,50 \%)\end{array}$ \\
\hline $\begin{array}{l}\text { Las clases así me motivan más a aprender contenidos sobre la unidad } \\
\text { y sobre las ciencias en general }\end{array}$ & $\begin{array}{l}22 / 24 \\
(91,70 \%)\end{array}$ & $\begin{array}{l}2 / 24 \\
(8,33 \%)\end{array}$ \\
\hline $\begin{array}{l}\text { Utilizar instrumentos científicos y hacer experimentos me gusta y } \\
\text { me ayudan a aprender }\end{array}$ & $\begin{array}{l}24 / 24 \\
(100 \%)\end{array}$ & $\begin{array}{l}0 / 24 \\
(0 \%)\end{array}$ \\
\hline $\begin{array}{l}\text { Me gusta más aprender con actividades colaborativas que siguiendo } \\
\text { el libro }\end{array}$ & $\begin{array}{l}21 / 24 \\
(87,50 \%)\end{array}$ & $\begin{array}{l}3 / 24 \\
(12,50 \%)\end{array}$ \\
\hline
\end{tabular}

Nota. No hubo puntuaciones para la categoría "No".

En ninguno de los casos los estudiantes prefieren aspectos ligados a la metodología tradicional en comparativa con su realización dentro de una metodología gamificada. No obstante, en algunas ocasiones se muestra indiferencia entre ambas formas de enseñanza, pero en ningún caso esta opción ha sido marcada por el alumnado en más de tres ocasiones. Con todo ello, se evidencia que la gamificación resulta atractiva al propio alumnado.

Esta visión, además, es claramente justificada por el alumnado en el espacio habilitado a continuación del cuestionario para resaltar todo aquello que estimaran oportuno. Esta información, unida a la visión cuantitativa descrita en los ítems, permite la triangulación de los datos (Cohen et al., 2000). En este espacio, los estudiantes brindaron información muy relevante a través de mostrar su perspectiva individual del proyecto. Las respuestas más frecuentes sugieren que los estudiantes encuentran varias ventajas relacionadas con el uso de la gamificación en clase. Destacando su marcado carácter lúdico y su capacidad para crear un ambiente de aprendizaje de mayor gusto para los estudiantes (Figura 7).

Figura 7

Conceptos más destacados y número de veces que aparecen en el test de motivación cumplimentado por el alumnado

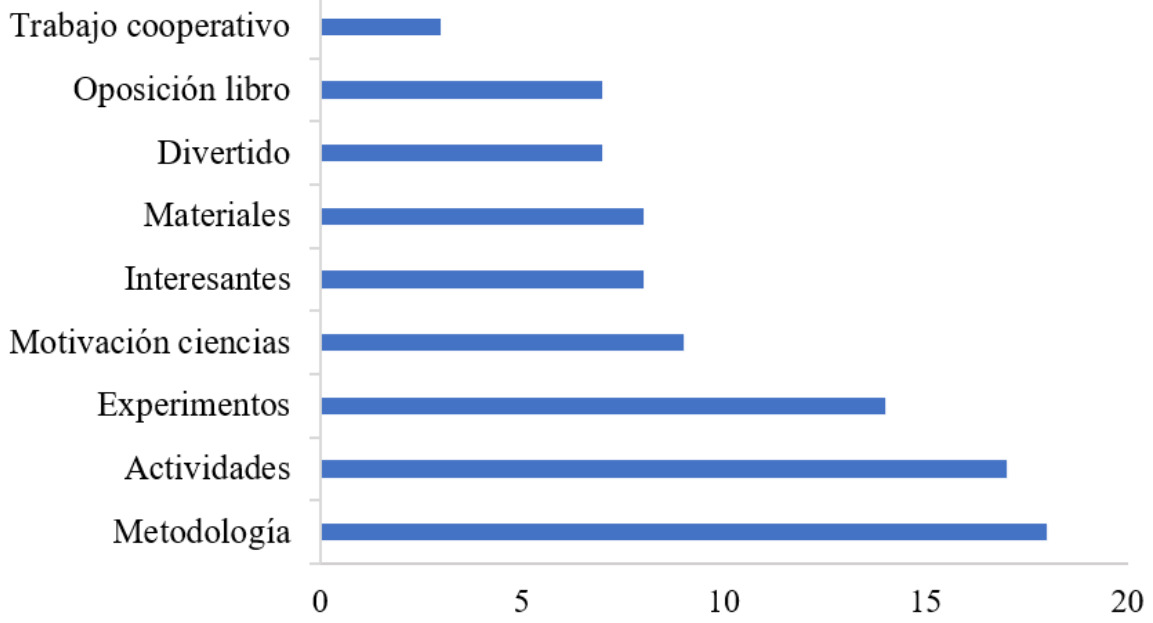


El alumnado muestra una gran afinidad con la metodología, materiales y actividades implementadas durante el proyecto: "así se interioriza mejor los conceptos" (Estudiante 3), destacando el gusto por los experimentos científicos realizados: "las clases así me parecen más divertidas, me motivan y eso hace que tengamos muchas ganas de aprender cosas nuevas, pienso que los experimentos nos han enseñado mucho" (Estudiante11). Además, los comentarios realizados por parte del alumnado resaltan lo divertido "ha sido una experiencia muy divertida, me han gustado todas las actividades" (Estudiante 9) e interesante de aprender así: "a mí no me gusta mucho estudiar y con las actividades y todo me ha motivado y me dan ganas de estudiar más" (Estudiante 21). Igualmente, destaca la oposición explicita que algunos realizan al estudio mediante el libro "Me lo he pasado muchísimo mejor que cuando seguimos el libro. Además, no tardaba tanto en hacer los deberes de Ciencias" (Estudiante 7). Finalmente, el alumnado también hace referencia al trabajo cooperativo "Me gusta más aprender así porque si no sé algo mis compañeros me ayudan” (Estudiante 18).

\section{Discusión}

A la vista de los resultados, se comprueba que el SEFG habilita al docente de un recurso pedagógico viable para la evaluación formativa dentro de proyectos de gamificación. Al mismo tiempo, dado que a través de esta metodología el alumnado adquiere el protagonismo en su aprendizaje, pues es quien construye su conocimiento de forma activa, el profesorado pasa de tener el rol de divulgador de conocimientos a ser facilitador del aprendizaje. Así, dado que delega ser el elemento transmisor de aprendizaje en favor de que el alumnado adquiera un rol activo, se habilita al docente a poder ir cumplimentando durante la sesión el propio diario de clase; lo que resulta más fiable que tras cada una de las sesiones y además supone una carga de trabajo adicional para el profesor. Si la herramienta es viable, pero supone una carga de trabajo añadida, el interés por su uso decrecerá (Jiménez-Gómez, 2019). Por ello, alcanzar la viabilidad de su uso dentro de la dinámica de clase en cada sesión es uno de los puntos fuertes alcanzados durante la presente investigación.

Con frecuencia encontramos entre la literatura la necesidad de formar al docente en nuevos recursos tecnológicos (Wu et al., 2019). Sin embargo, la investigación en la renovación eficaz de recursos tradicionales suele despertar poco interés. No obstante, se ha comprobado que la adaptación de recursos a los nuevos planteamientos metodológicos resulta más atractiva para el profesorado; pues ya conocen su uso (Constantine y Jung, 2019). Dado que la inclusión del SEFG no requiere la utilización de ninguna aplicación poco común en el ámbito educativo, la aceptación en su uso por parte del profesorado es mucho más factible en la línea de lo comentado por autores como Jiménez-Gómez (2019).

Centrándonos en su utilidad en el proceso de evaluación, los objetivos marcados en el estudio han sido confirmados. Se ha evidenciado la eficacia del SEFG para la evaluación del proceso de aprendizaje de forma continua en la línea de los resultados alcanzados por otros autores (Paek y Fulton, 2017). Esto, además, se ha contrastado en la evaluación de aspectos específicos del proceso de enseñanza. En referencia exclusiva a la adquisición de conocimientos, podemos afirmar que el SEFG permite al docente el correcto seguimiento del logro académico; sin necesidad de recurrir a pruebas evaluativas sumativas al finalizar el proyecto.

Igualmente, mientras que con el sistema de evaluación tradicional por medio de examen no es hasta el final del proceso de enseñanza cuando se conoce el logro alcanzado por el alumnado una vez fechadas y corregidas las pruebas, con el SEFG existe una retroalimentación diaria de cómo se está produciendo el aprendizaje gracias a los puntos de experiencia asociados a los retos realizados (Chou, 2019; Zichermann y Cunnigham, 2011). De igual modo, este estudio ha confirmado la utilidad del uso del SEFG en relación al objetivo dos: la evaluación formativa de aspectos transversales de gran transcendencia durante el proceso de enseñanza-aprendizaje como son el interés, actitud y trabajo cooperativo.

Dado que el alumnado podía ver gracias al portal web cuántos puntos tenía en cada uno de los aspectos analizados, era plenamente consciente de cómo estaba siendo su trabajo en clase y podía tomar decisiones durante el propio proyecto para mejorar. De este modo, el alumnado no era mero receptor de esta información al finalizar el proyecto, como sí sucede en la evaluación sumativa tradicional, sino que era partícipe del mismo. Autores como Nicol y Miligan (2006) demuestran la eficacia de estos procesos de 
autorregulación y la retroalimentación continua al poder acceder a esta información durante el propio proceso de aprendizaje y poder modificar su trayectoria. El sistema por recompensas típico de los videojuegos adaptado al presente proyecto hace que el interés del alumnado por estos proyectos mejore (Fiellin et al., 2014; Prestopnik y Tang, 2015). Los alumnos, al ver que su actuación tiene repercusión en el número de puntos alcanzados, aprecian un sentido de progresión y de recompensa al esfuerzo que les incentiva a participar de forma correcta. Esta motivación del alumnado queda contrastada gracias al objetivo tres de la investigación y la opinión personal del alumnado hacia el proyecto.

El alumnado ha mostrado gran satisfacción con las actividades, retos y experimentos realizados en clase; confirmando la motivación que la gamificación puede propiciar en el contexto educativo (Kapp, 2012). Al mismo tiempo, valoran positivamente la realización de tareas por medio del trabajo cooperativo con sus compañeros. Puesto que la resolución de problemas y el trabajo por competencias tales como aprender a aprender o la cooperación entre alumnos son elementos curriculares prioritarios actualmente (Díaz y Troyano, 2013; Jagušt et al., 2018; LOMCE, 2013), que el alumnado se encuentre cómodos con esta metodología lúdica supone un refuerzo positivo a los resultados encontrados. Finalmente, dado que el alumnado muestra actualmente poco interés por los contenidos de ciencias (Toma et al., 2019), esta mejora de la motivación en clase supone dar respuesta a una de las grandes necesidades de la didáctica de las ciencias (Solbes, 2011).

\section{Conclusiones}

Uno de los principales problemas a los que se enfrenta la innovación educativa es cómo ofrecer alternativas válidas a la evaluación tradicional sumativa (Zepeda-Hernández et al., 2016). Por ello, el presente estudio centró su objeto de investigación en el análisis de un sistema evaluativo formativo gamificado (SEFG) por medio de la utilización de puntos de experiencia recogidos en el tradicional diario de clase adaptado al formato tecnológico. Durante 14 sesiones, correspondientes a la realización de una unidad didáctica completa en Ciencias Naturales, se ha implementado la gamificación junto a este sistema con el objeto de analizar la viabilidad de su uso para alcanzar la evaluación formativa del alumnado de todos los aspectos ligados al proceso de enseñanza.

A la vista de los resultados, podemos afirmar que se han confirmado los objetivos de investigación planteados. La adopción de la mecánica evaluativa por puntos de experiencia típica de los videojuegos (Fiellin et al., 2014), adaptados al contexto educativo, dota al maestro de la información necesaria para la evaluación continua de su alumnado. El objetivo del estudio referido a poder analizar el rendimiento académico, y aspectos transversales (trabajo cooperativo, realización de tareas e interés), ha sido confirmado gracias al empleo del SEFG. Por tanto y pese a que la investigación educativa no suele prestar la atención necesaria a la innovación evaluativa de estos aspectos transversales, podemos afirmar que el SEFG permite estructurar de una forma continua y concisa cómo evaluar todos los aspectos acontecidos en el aula tales como la resolución de problemas, la cooperación y la reflexión crítica (Díaz y Troyano, 2013; Jagušt et al., 2018; Labrador y Villegas, 2016).

De este modo, no solo se dota al docente de un nuevo sistema de evaluación alternativo, sino que, además, dada la metodología lúdica-activa implementada definida por Marczewski (2013) como la gamificación profunda, se refuerza el interés del alumnado por aprender al adoptarse dentro del proceso de aprendizaje componentes, mecánicas y dinámicas del juego (Nistor e Iacob, 2018). Algo destacable dado el actual declive actitudinal hacia las ciencias (Pečiuliauskienè y Belakoz, 2019; Toma et al., 2019). Esta mejora de la motivación es también afirmada por el propio alumnado, pues apuntan que prefiere esta dinámica de clase en la que participan de forma activa en su aprendizaje, realizando experimentos y resolviendo retos, en comparación a la metodología tradicional en la que son oyentes pasivos, y en la que el libro de texto y la realización de un examen al finalizar el proceso cobran gran importancia (De Ory y Ruiz, 2011). Por ello, continuar en esta línea de actuación educativa resultaría interesante para alcanzar una innovación pedagógica completa: no solo desde el punto de vista evaluativo, sino también metodológico. 


\section{Limitaciones y futuros estudios}

Dado el actual momento de cambio pedagógico en el que nos encontramos debido a las nuevas demandas sociales, estudios como el aquí presente abren la puerta a nuevos planteamientos evaluativos que complementen a investigaciones de gran envergadura como, por ejemplo, el impacto de la gamificación en el entorno educativo. Si bien es cierto que el proyecto tuvo lugar durante una unidad didáctica completa, se hacen necesarias investigaciones que cubran un mayor periodo de tiempo (p. ej., un curso académico). Además, otra de las debilidades del estudio es que este ha sido realizado exclusivamente con una única clase, por lo que la muestra de participantes es reducida. A la vista de los resultados, el SEFG se presenta como una alternativa prometedora del proceso evaluativo durante dinámicas de aprendizaje lúdicas. Por ello, se hace necesario continuar en esta línea de investigación, ampliando el número de participantes y la duración de las intervenciones y temáticas gamificadas, con el objeto de confirmar la posibilidad de generalizar las conclusiones aquí presentadas.

Asimismo, durante el proyecto han surgido nuevas líneas de investigación a explorar en el futuro. En vista del interés del alumnado por la realización de los retos extra de carácter voluntario, un análisis detallado de las causas que generan este interés puede ser altamente informativa acerca de a qué obedece el actual desinterés de los estudiantes por los contenidos de ciencias. Por este motivo, profundizar en la percepción del alumnado acerca de metodologías puede permitir mayor rotundidad en los resultados presentados. Por ello, en futuros estudios pretendemos ampliar el test motivacional con preguntas específicas sobre la afinidad del alumnado con este tipo de metodologías. Por otro lado, dada la importancia de la opinión del profesorado acerca de la efectividad y viabilidad de los instrumentos de evaluación, en futuros estudios plantemos conocer la visión de los docentes hacia el SEFG, y su uso, lo que podría suscitar la necesidad de nuevos planteamientos metodológicos o de cambios en la propia herramienta.

\section{Referencias}

Alsawaier, R. S. (2018). The effect of gamification on motivation and engagement. The International Journal of Information and Learning Technology, 35(1), 56-79. https://doi.org/10.1108/IJILT-02-2017-0009

Álvarez Méndez, J. M. (2009). Evaluar el aprendizaje en una enseñanza centrada en competencias. En J. Gimeno Sacristán (Comp.), Educar por competencias, ¿qué hay de nuevo? (pp. 206-233). Madrid: Morata, 2a Edición

Ascendio Peralta, C. (2016). Adecuación de la planeación didáctica como herramienta docente en un modelo universitario orientado al aprendizaje. REICE. Revista Iberoamericana sobre Calidad, Eficacia y Cambio en Educación, 14(3), 109-130. https://doi.org/10.15366/reice2016.14.3.006

Bai, S., Hew, K. F. y Huang, B. (2020). Does gamification improve student learning outcome? Evidence from a meta-analysis and synthesis of qualitative data in educational contexts. Educational Research Review, 30, 100322. https://doi.org/10.1016/j.edurev.2020.100322

CEIP Deitania Comarcal. (2018). El compromiso de la galaxia Deitania. https:/www.edu.xunta.gal/centros/ iesblancoamorculleredo/aulavirtual2/pluginfile.php/29962/mod_resource/content/1/Gu\%C3\%ADa\%20 did\%C3\%A1ctica\%20de\%20una\%20escape\%20room\%20para\%203.\%20\%C2\%BA\%20de\%20E.\%20 Primaria\%20\%28PAGT\%29.pdf

CEIP Reino de Murcia. (2018). Pequeños detectives al rescate. http://www.colegioreinodemurcia.com/2018/05/pequenos-detectives-al-rescate-durante.html

Chou, Y. K. (2019). Actionable gamification: Beyond points, badges, and leaderboards. Packt Publishing Ltd.

Cohen, L., Manion, L. y Morrison, K. (2000). Research methods in education. Routledge.

Colegio Valle del Miro. (2018). Operación beijerinck. https://www.colegiovalledelmiro.es/blog/post/operacionbeijerinck-mejor-experiencia-gamificacion-simo-2018/

Constantine, A. y Jung, K. G. (2019). Using digital science notebooks to support elementary student learning: Lessons and perspectives from a fifth-grade science classroom. Contemporary Issues in Technology and Teacher Education, 19(3), 373-412. 
De Ory, M. y Ruiz, V. (2011). La evaluación en el aula de primaria. Factor clave para el aprendizaje de las ciencias y las matemáticas. Revista Eureka sobre Enseñanza y Divulgación de las Ciencias, 8(2), 212-220.

Díaz, J. y Troyano, Y. (2013, 7 de mayo). El potencial de la gamificación aplicado al ámbito educativo [Comunicación]. III Jornadas de Innovación Docente. Sevilla, España.

Feijóo, J. y Hernantes, D. (2017). Lean Gamification. Crea productos exitosos y motiva el deseo de tus clientes. Fundación Confemetal.

Fiellin, L. E., Hieftje, K. D. y Duncan, L. R. (2014). Videogames, here for good. Pediatrics, 134(5), 849-851. https://doi.org/10.1542/peds.2014-0941

Flecha, R. y Molina, S. (2015). Actuaciones educativas de éxito como base de una política educativa basada en evidencias. Avances en Supervisión Educativa, 32, 1-18. https://doi.org/10.23824/ase.v0i23.23

Hamari, J., Koivisto, J. y Sarsa, H. (2014). Does gamification work? A literature review of empirical studies on gamification. HICSS, 14, 3025-3034.

Hattie, J. (2009). Visible learning. A synthesis of over 800 meta-Analyses relating to achievement. Routledge.

Huang, R., Ritzhaupt, A. D., Sommer, M., Zhu, J., Stephen, A., Valle, N., Hampton, J. y Li, J. (2020). The impact of gamification in educational settings on student learning outcomes: A meta-analysis. Educational Technology Research and Development, 68(4), 1875-1901. https://doi.org/10.1007/s11423-020-09807-z

Hursen, C. y Bas, C. (2019). Use of gamification applications in science education. International Journal of Emerging Technologies in Learning, 14(1), 4-23. https://doi.org/10.3991/ijet.v14i01.8894

Instituto Nacional de Evaluación Educativa. (2019). Informe PISA 2018. Informe español. INEE.

Jagušt, T., Botički, I. y So, H. J. (2018). Examining competitive, collaborative and adaptive gamification in young learners' math learning. Computers \& Education, 125, 444-457. https://doi.org/10.1016/j.compedu.2018.06.022

Jiménez-Gómez, M. A. (2019). ¿Pueden las TIC mejorar la evaluación? e-CO. Revista Digital de Educación y Formación del Profesorado, 16, art 7.

Kapp, K. (2012). The gamification of learning and instruction: Game-based methods and strategies for training and education. John Wiley \& Sons.

Labrador, E. y Villegas, E. (2016). Unir gamificación y experiencia de usuario para mejorar la experiencia docente. Revista Iberoamericana de Educación a Distancia, 19(2), 125-142. https://doi.org/10.5944/ried.19.2.15748

López-Lozano, L. y Solís, E. (2015). Los estudiantes de magisterio frente a la evaluación. En N. Gonzalez, I. Salcines y E. García (Coords.), Tendencias emergentes en evaluación formativa y compartida en docencia: El papel de las nuevas tecnologías (pp. 949-958). Editorial de la Universidad de Cantabria.

Lyons, T. y Quinn, F. (2010). Choosing science. Understanding the declines in senior high school science enrolments. SiMERR.

Marczewski, A. (2013). Gamification: A simple introduction and a bit more. Kindle.

Martin, M. O., Mullis, I. V., Foy, P. y Stanco, G. M. (2012). TIMSS 2011 international results in science. International Association for the Evaluation of Educational Achievement.

Navarro, G. M. (2017). Tecnologías y nuevas tendencias en educación: aprender jugando. El caso de Kahoot. Revista de Ciencias Humanas y Sociales, 83, 252-277.

Nicol, D. y Milligan, C. (2006). Rethinking technology-supported assessment practices in relation to the seven principles of good feedback practice. En C. Bryan y K. Clegg (Eds.), Innovative Assessment in Higher Education (pp. 247-268). Taylor and Francis Group.

Nistor, G. y Iacob, A. (2018, 28 de septiembre). The advantages of gamification and game-based learning and their benefits in the development of education [Comunicación]. The International Scientific Conference eLearning and Software for Education. Bucharest, Rumanía.

Nolan,J.y McBride, M. (2014). Beyond gamification: Reconceptualizing game-based learning in early childhood environments. Information, Communication \& Society, 17(5), 594-608. https://doi.org/10.1080/1369118X.2013.808365 
Ortiz-Colón, A. M., Jordán, J. y Agredal, M. (2018). Gamificación en educación: Una panorámica sobre el estado de la cuestión. Educação e Pesquisa, 44, 1-17. https://doi.org/10.1590/s1678-4634201844173773

Paek, S. y Fulton, L. (2017). Digital science notebooks: perspectives from an elementary classroom teacher. Journal of Computers in Mathematics and Science Teaching, 36(4), 361-374.

Pečiuliauskienè, P. y Belakoz, A. (2019). School students' motivation for learning sciences: How is it influenced by self-confidence and inquiry-based approach? Pedagogy, 134(2), 121-134. https://doi.org/10.15823/p.2019.134.8

Piaget, J. (1952). The origins of intelligence in children. International Universities Press.

Porlán, R. (2008). El diario de clase y el análisis de la práctica. Averroes, 8, 1-8.

Prestopnik, N. R. y Tang, J. (2015). Points, stories, worlds, and diegesis: Comparing player experiences in two citizen science games. Computers in Human Behavior, 52, 492-506. https://doi.org/10.1016/j.chb.2015.05.051

Rowcliffe, S. (2004). Storytelling in science. School Science Review, 86(314), 121.

Sá, J. (2002). Diary writing: An interpretative research method of teaching and learning. Educational Research and Evaluation, 8(2), 149-168. https://doi.org/10.1076/edre.8.2.149.3858

Sedeño, A. (2010). Videojuegos como dispositivos culturales: Las competencias espaciales en educación. Comunicar, 34, 183-189. https://doi.org/10.3916/C34-2010-03-18

Solbes, J. (2011). ¿Por qué disminuye el alumnado de ciencias? Alambique: Didáctica de las ciencias experimentales, 67, 53-61.

Taylor, S. y Bogdan, R. (2000). Introducción a los métodos cualitativos. Paidós.

Toma, R., Ortiz-Revilla, J. y Greca, I. M. (2019). ¿Qué actitudes hacia la ciencia posee el alumnado de Educación Primaria que participa en actividades científicas extracurriculares? Ápice. Revista de Educación Científica, 3(1), $55-69$.

Vázquez, A. y Manassero, M. A. (2008). El declive de las actitudes hacia la ciencia de los estudiantes: un indicador inquietante para la educación científica. Revista Eureka sobre Enseñanza y Divulgación de las Ciencias, 5(3), 274292.

Wang, A. I. y Lieberoth, A. (2016, 3 de noviembre). The effect of points and audio on concentration, engagement, enjoyment, learning, motivation, and classroom dynamics using Kahoot [Comunicación]. European Conference on Games Based Learning. Reading, UK.

Werbach, K. y Hunter, D. (2015). The gamification toolkit: dynamics, mechanics, and components for the win. Wharton School Press.

Wu, B., Hu, Y. y Wang, M. (2019). Scaffolding design thinking in online STEM preservice teacher training. British Journal of Educational Technology, 50(5), 2271-2287. https://doi.org/10.1111/bjet.12873

Zepeda-Hernández, S., Abascal-Mena, R. y López-Ornelas, E. (2016). Integración de gamificación y aprendizaje activo en el aula. Ra Ximhai, 12(6), 315-325.

Zichermann, G. y Cunningham, C. (2011). Gamification by design: Implementing game mechanics in web and mobile apps. O’Reilly.

\section{Breve CV de los/as autores/as}

\section{Sergio Tirado-Olivares}

Graduado en Maestro/a en Educación Primaria y premio extraordinario en el Máster en Investigación e Innovación Educativa de la Universidad de Castilla-La Mancha. Personal contratado de I+D en el grupo de investigación consolidado LabinTic (Laboratorio de Integración de las Tecnologías en el Aula). Sus líneas de investigación se centran en la aplicación de contextos lúdico-activos de aprendizaje con alumnado de Educación Primaria y docentes en formación, la aplicación de metodologías activas como la gamificación, el desarrollo de nuevos planteamientos de evaluación formativos dentro de estas dinámicas 
y el fomento de las vocaciones hacia disciplinas STEAM. Actualmente, cuenta con un artículo publicado como primer autor en la Revista Española de Educación Comparada indexada en Scopus. Email: sergio. tirado@uclm.es

ORCID ID: https://orcid.org/0000-0002-8557-5115

\section{José Antonio González-Calero}

Profesor Titular de Universidad en el área de Didáctica de las Matemáticas del departamento de Matemáticas de la Universidad de Castilla-La Mancha. Hasta la fecha, tiene 1 sexenio de investigación y ha publicado 37 artículos de investigación (17 indexados en JCR) y ha participado en el equipo de investigación de 1 proyecto internacional, 6 nacionales y múltiples de carácter local o regional. Como investigador actualmente dirige un proyecto de investigación de carácter regional y también codirige el grupo de investigación LabinTic (Laboratorio de Integración de las Tecnologías en el Aula). En el ámbito docente, ha dirigido dos proyectos de innovación competitivos de la Universidad de Castilla-La Mancha y participado en otros 10. Email: jose.gonzalezcalero@uclm.es

ORCID: https://orcid.org/0000-0003-0842-8151

\section{Ramón Cózar-Gutiérrez}

Profesor Titular de Universidad en el departamento de Historia de la Facultad de Educación de Albacete de la Universidad de Castilla-La Mancha. Tiene concedidos 3 sexenios, 2 de investigación y 1 de transferencia. Cuenta con más de 150 publicaciones, de las que 8 son monografías, 36 están indexadas en Web of Science y 19 en Scopus. Ha participado en el equipo de investigación de 1 proyecto internacional, 4 nacionales y más de 30 de carácter regional, desempeñando el rol de investigador principal en al menos una veintena. Codirige el grupo de investigación consolidado de la UCLM, LabinTic. Laboratorio de Integración de la tecnología en las aulas. Desde 2010 es director de Ensayos. Revista de la Facultad de Educación de Albacete, indexada en ESCI de WOS. Email: ramon.cozar@uclm.es

ORCID: https://orcid.org/0000-0001-8255-6376

\section{Rosa M. Toledano}

Investigadora y profesora asociada del Departamento de Química-Física en la Facultad de Educación de Albacete de la Universidad de Castilla-La Mancha desde 2016. Ha participado como miembro del equipo de investigación en 1 proyecto internacional, 5 nacionales y 3 regionales, además de en 5 proyectos de innovación docente. Cuenta con más de 25 publicaciones (19 artículos indexados en JCR), es coautora de 4 patentes de invención. Su principal investigación se centra en el desarrollo de métodos analíticos por técnicas cromatográficas multidimensionales. También desarrolla una línea de investigación centrada en la enseñanza-aprendizaje de las ciencias experimentales. Es coordinadora del Grado en Educación Primaria (Titulación en Lengua Inglesa). Email: rosa.toledano@uclm.es

ORCID: https://orcid.org/0000-0003-2376-9341 